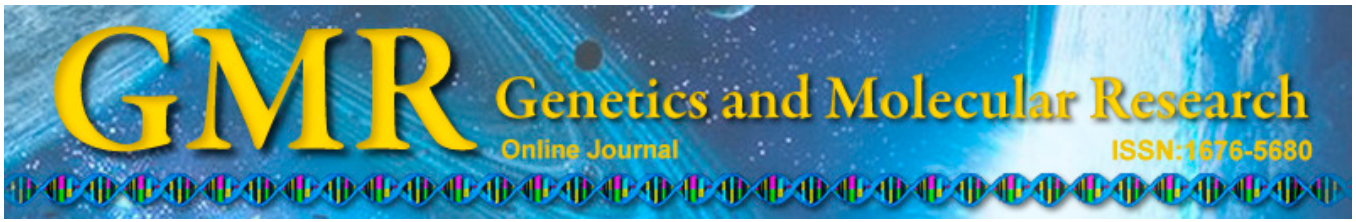

\title{
Macrophage proinflammatory response to the titanium alloy equipment in dental implantation
}

\author{
X. Chen, H.S. Li, Y. Yin, Y. Feng and X.W. Tan \\ Department of Stomatology, The General Hospital of the Air Force, \\ Beijing, China \\ Corresponding author: Xin Chen \\ E-mail: xinchenbei@sina.com
}

Genet. Mol. Res. 14 (3): 9155-9162 (2015)

Received January 28, 2015

Accepted April 29, 2015

Published August 7, 2015

DOI http://dx.doi.org/10.4238/2015.August.7.25

\begin{abstract}
Titanium alloy and stainless steel (SS) had been widely used as dental implant materials because of their affinity with epithelial tissue and connective tissue, and good physical, chemical, biological, mechanical properties and processability. We compared the effects of titanium alloy and SS on macrophage cytokine expression as well as their biocompatibility. Mouse macrophage RAW264.7 cells were cultured on titanium alloy and SS surfaces. Cells were counted by scanning electron microscopy. A nitride oxide kit was used to detect released nitric oxide by macrophages on the different materials. An enzyme linked immunosorbent assay was used to detect monocyte chemoattractant protein-1 levels. Scanning electron microscopy revealed fewer macrophages on the surface of titanium alloy (48.2 \pm $\left.6.4 \times 10^{3} \mathrm{cells} / \mathrm{cm}^{2}\right)$ than on SS $\left(135 \pm 7.3 \times 10^{3} \mathrm{cells} / \mathrm{cm}^{2}\right)$. The nitric oxide content stimulated by titanium alloy was $22.5 \mu \mathrm{M}$, which was lower than that stimulated by SS $(26.8 \mu \mathrm{M})$, but the difference was not statistically significant $(\mathrm{P}=0.07)$. The level of monocyte chemoattractant protein-1 released was significantly higher in the SS group (OD value $=0.128)$ than in the titanium alloy group $(\mathrm{OD}$ value $=0.081)(\mathrm{P}=$
\end{abstract}


$0.024)$. The transforming growth factor- $\beta 1 \mathrm{mRNA}$ expression levels in macrophages after stimulation by titanium alloy for 12 and $36 \mathrm{~h}$ were significantly higher than that after stimulation by $\mathrm{SS}(\mathrm{P}=0.31$ and 0.25 , respectively). Macrophages participate in the inflammatory response by regulating cytokines such as nitric oxide, monocyte chemoattractant protein-1, and transforming growth factor- $\beta 1$. There were fewer macrophages and lower inflammation on the titanium alloy surface than on the SS surface. Titanium alloy materials exhibited better biological compatibility than did SS.

Key words: Dental implantation; Macrophage; Titanium alloy

\section{INTRODUCTION}

Dental implant materials should have good biocompatibility, including no cytotoxicity, irritation, immune reaction, allergic reaction, genetic toxicity, and carcinogenicity, and can coexist within the body (Manzanares et al., 2001). Dense oxide film on the titanium surface can combine with substrate over time to inhibit the metal ion activity. This allows the titanium metal to have stable biological properties (Steflik et al., 1999). Titanium and titanium alloy have been widely used in dental implantation, such as for removable partial denture, complete denture, and tooth defect repair, because of its good physical and chemical properties, biological properties, mechanical properties, and processability. It is currently the universal dental implant material used worldwide. However, immune rejection can occur after dental implant materials are placed into the body. Neutrophil and macrophage accumulation can appear in the tissue around the implant materials for wound repair, leading to a local inflammatory response (Dalal et al., 2012; Cachinho et al., 2013). Macrophages are the main phagocytes in the early stages of inflammation when the body contacts the implant material. Previous studies have revealed that different implant materials have different biocompatibilities, leading to different macrophage responses and outcomes. This can produce varying inflammatory reactions and healing processes (De Nardo et al., 2012; Yazici et al., 2013). RAW264.7 cells are mononuclear macrophage leukemia cells derived from male Abelson murine tumors induced by the leukemia virus in mice. This type of macrophage is easily cultivated and proliferates quickly. In this study, we cultured the mouse mononuclear cell line RAW264.7 on the surface of titanium alloy or medical stainless steel (SS) implant material to examine the macrophage proinflammatory response to different types of materials implanted in the body. We investigated the different functions of titanium alloy and SS on macrophage activity to provide a foundation for further study.

\section{MATERIAL AND METHODS}

\section{Materials}

\section{Reagents}

DMEM medium was from Beijing Gaoke Henghui Co. (Beijing, China). Fetal bovine serum was from Sigma (St. Louis, MO, USA). The NO kit was from HUYU Biological Tech- 
nology Co., Ltd. (Shanghai, China). Monocyte chemoattractant protein-1 (MCP-1) monoclonal antibody was from BioLegend (San Diego, CA, USA). Sodium bicarbonate, anhydrous ethanol, ether, acetone, formaldehyde, phosphate-buffered saline were prepared in our lab.

\section{Experimental apparatus}

In this study, we used a spectrophotometer (B-800; Metash, Shanghai, China), realtime fluorescent quantitative polymerase chain reaction (PCR) amplifier (Bio-Tek, Winooski, VT, USA), $\mathrm{CO}_{2}$ incubator (Thermo Scientific, USA), Leica inverted fluorescence microscope (Leica, Wetzlar, Germany), microplate reader (Bio-Tek), centrifuge (TDD5K; Changsha Dongwang Experimental Instrument Co., Ltd., Hunan, China), critical drying apparatus (AUTOSamdri-815-b; Keyang Co., Ltd., Shanghai, China), environmental scanning electron microscope (KYKY-EM6000C; KYKY Technology Development Co., Ltd., Beijing, China), ultrasonic cleaning machine (KQ-500E, Ziyi Reagent, Shanghai, China), autoclave (YXQLS-100SII; Boxum, Shanghai, China), real-time PCR kit (Takara, Shiga, Japan), primers (Generay, Shanghai, China), titanium alloy, SS, and the mouse mononuclear macrophage cell line RAW264.7. Primer sequences are listed in Table 1.

\begin{tabular}{ll}
\multicolumn{1}{c}{ Table 1. Primer sequences. } \\
\hline Gene & Sequence (5'-3') \\
\hline TGF- $\beta 1$ & Forward: CTGTCCAAACTAAGGCTCGC \\
MCP-1 & Reverse: CGTCAAAAGACAGCCACTCA \\
& Forward: CTCACCTGCTGCTACTCATTC \\
GAPDH & Reverse: GCTTGAGGTGGTTGTGGAAAA \\
& Forward: TCACTCAAGATTGTCCAGCAA \\
\hline
\end{tabular}

\section{Methods}

\section{Materials preparation}

Titanium alloy and SS were prepared be $15 \mathrm{~mm}$ in diameter and $1 \mathrm{~mm}$ in thickness. The materials were ultrasonic-cleaned in acetone, ethanol, and distilled water in sequence. Materials were then sterilized in an autoclave.

\section{Cell culture}

The RAW264.7 cell line was maintained in high-glucose DMEM medium supplement with $10 \%$ fetal bovine serum in a humidified atmosphere with $5 \% \mathrm{CO}_{2}$ at $37^{\circ} \mathrm{C}$.

\section{Immunofluorescent staining}

Macrophages were washed with phosphate-buffered saline 3 times, fixed with $4 \%$ formaldehyde for 20-30 min, and stained with 4',6-diamidino-2-phenylindole for $10 \mathrm{~min}$. The plate was then observed under a microscope. 


\section{Scanning electron microscope observation}

After the macrophages were cultured for $72 \mathrm{~h}$, the material surface was observed and photographed under scanning electron microscopy after spraying.

\section{Nitric oxide $(\mathrm{NO})$ release detection}

The sample supernatant was collected. After staining, the absorption luminosity value was read using a microplate reader according to the manufacturer instructions.

\section{MCP-1 release detection}

An enzyme-linked immunosorbent assay was used to detect MCP-1 content according to the manufacturer instructions.

\section{Real-time PCR}

Real-time PCR was conducted to detect transforming growth factor (TGF)- $\beta 1$ expression in cells at 12,36 , and $60 \mathrm{~h}$.

\section{Statistical analysis}

All statistical analyses were performed using the SPSS 11.5 software (SPSS, Inc., Chicago, IL, USA). Differences in macrophage number, NO expression, MCP-1 mRNA expression, and TGF- $\beta 1$ mRNA expression were analyzed using the Student $t$ test. P values $<$ 0.05 were considered to be statistically significant.

\section{RESULTS}

\section{Macrophage comparison}

As shown in Figure 1, macrophage number on the titanium alloy surface was lower than that on the SS surface after culture for $72 \mathrm{~h}$.
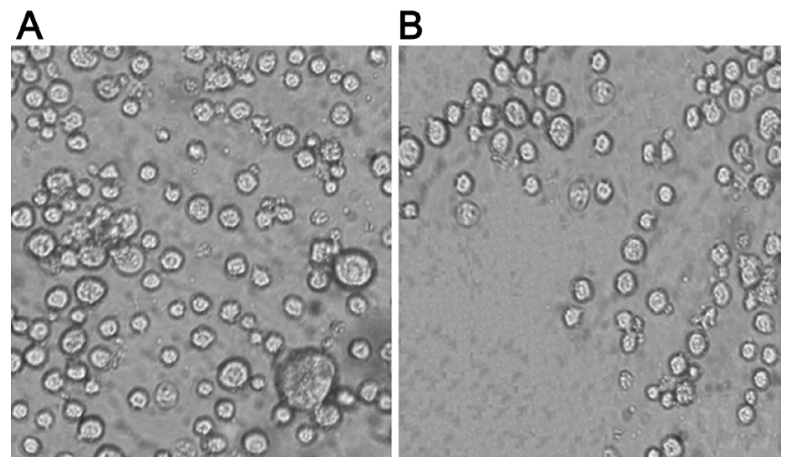

Figure 1. Macrophage comparison. A. Macrophage on titanium alloy (500X). B. Macrophage on SS (500X). 
The number of macrophages on the titanium alloy surface was $135 \pm 7.3 \times 10^{3}$ cells/ $\mathrm{cm}^{2}$, while the number of macrophages was significantly lower on the SS surface (48.2 \pm 6.4 $\mathrm{x} 10^{3}$ cells $\left./ \mathrm{cm}^{2}\right)(t=19.20, \mathrm{P}=0.02)($ Table 2$)$.

Table 2. Number of macrophages on the surface of titanium alloy and SS.

\begin{tabular}{lcc}
\hline & \multicolumn{2}{c}{ Macrophage number $\left(\mathrm{x} 10^{3} \mathrm{cells} / \mathrm{cm}^{2}\right)$} \\
\cline { 2 - 3 } & Titanium alloy & SS \\
\hline SEM (500X) & $48.2 \pm 6.4$ & $135 \pm 7.3$ \\
\hline
\end{tabular}

\section{NO release comparison}

The NO content stimulated by titanium alloy was $22.5 \mu \mathrm{M}$ and by SS was $26.8 \mu \mathrm{M}$, but the difference was not statistically significant $(\mathrm{P}=0.07)$ (Figure 2$)$.

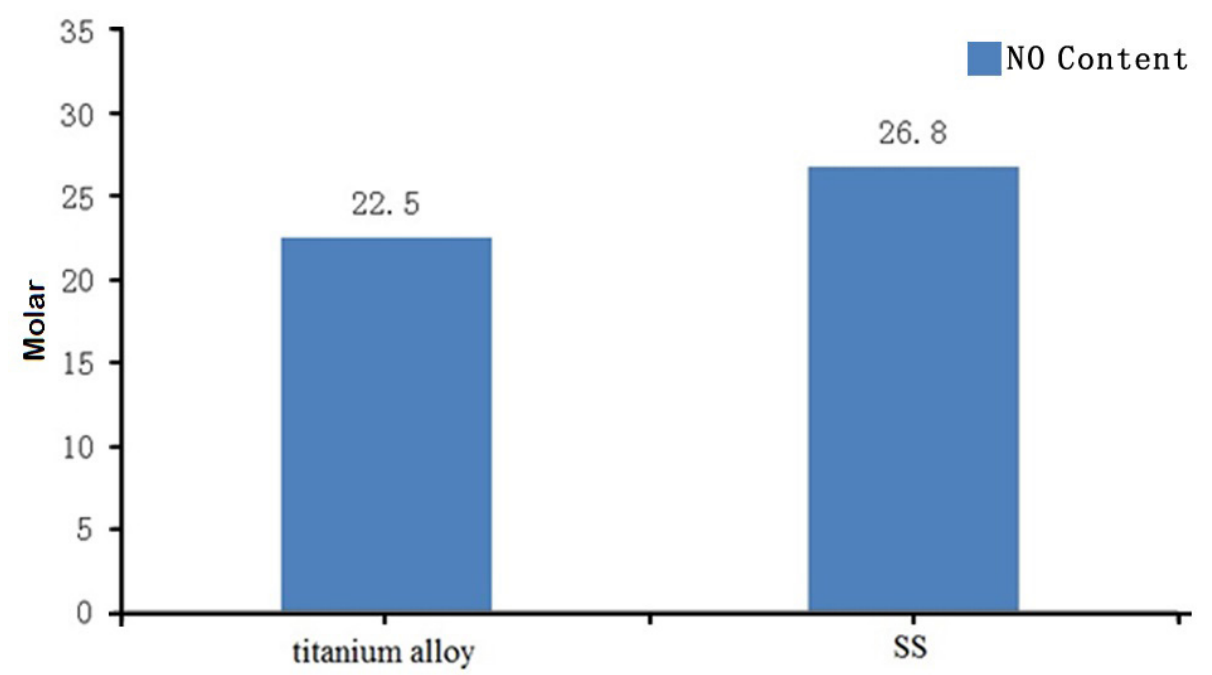

Figure 2. NO release comparison.

\section{MCP-1 mRNA expression comparison}

$\mathrm{MCP}-1$ released in the SS group $(\mathrm{OD}$ value $=0.128)$ was significantly higher than that in the titanium alloy group $(\mathrm{OD}$ value $=0.081)(\mathrm{P}=0.024)$ (Figure 3$)$.

\section{TGF- $\beta 1$ mRNA expression comparison}

TGF- $\beta 1$ mRNA relative expression levels in macrophages on the titanium alloy surface were $0.86,2.06$, and 1.72 at 12,36 , and $60 \mathrm{~h}$, while expression levels in macrophages on the SS surface were $0.56,1.05$, and 2.13 , respectively (Figure 4A). TGF- $\beta 1$ mRNA expression levels in macrophages after stimulation on titanium alloy for 12 and $36 \mathrm{~h}$ were significantly higher than that macrophages on SS ( $\mathrm{P}=0.31$ and 0.25 , respectively) (Figure $4 \mathrm{~B})$. 
A

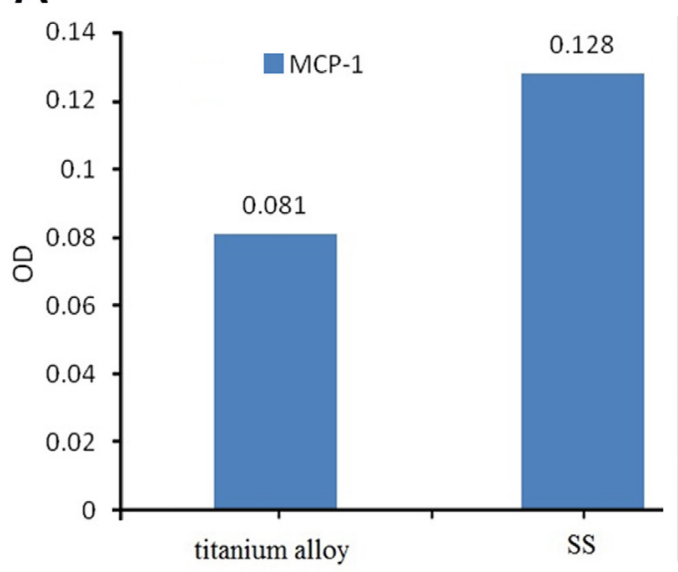

B

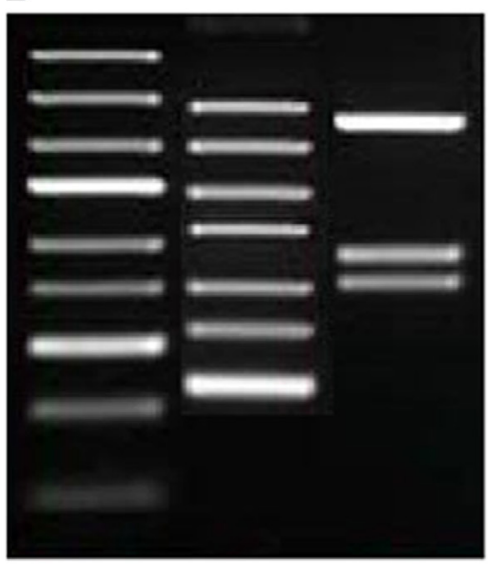

Figure 3. MCP-1 mRNA expression comparison. A. MCP-1 content comparison. B. MCP-1 electrophoretogram.

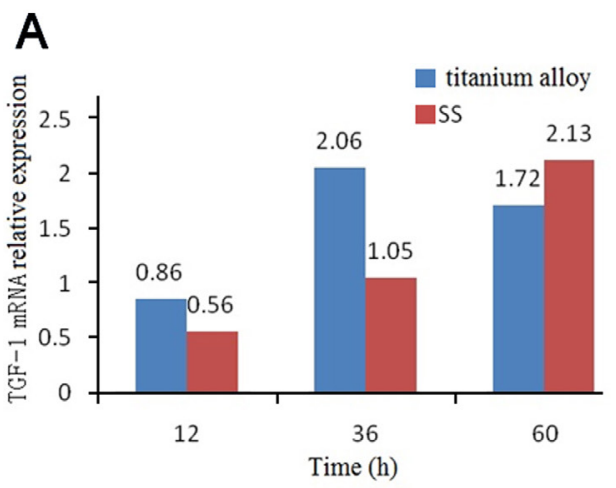

B

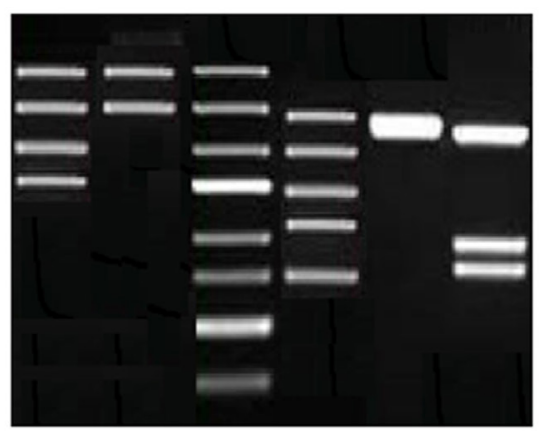

Figure 4. TGF- $\beta 1$ and mRNA A. TGF- $\beta 1$ mRNA expression comparison. B. TGF- $\beta 1$ mRNA electrophoretogram.

\section{DISCUSSION}

Dental materials can cause inflammation in adjacent tissue, and the body can remove pathogens to organize the gradual re-growing and wound healing process (Lee et al., 2012; Jiang et al., 2013). Various inflammatory mediators in the blood direct mononuclear cells towards the wounded tissue for further differentiation into macrophages after the placement of biological materials. Activated macrophages clean the damaged and dead cells and bacteria through inflammatory processes (Arango Duque and Descoteaux, 2014; Hu et al., 2014). Additionally, numerous biologically active substances can guide tissue repair. The type and number of cytokines released by macrophages may affect the stability and histocompatibility of the biological materials during inflammation. Understanding the effects of titanium alloy and SS on macrophage activity can lead to the development of methods for improving the interaction between implant materials and tissue interfaces, which may assist in the selection of appropriate implant materials (Malik et al., 2002; Jin et al., 2014). 
In this study, we acquired photos of titanium alloy and SS under scanning electron microscopy and found a larger number of macrophages on the SS surface. This indicates that the titanium alloy showed a lower degree of macrophage activation and better biocompatibility.

NO plays an important role in the immune system in resisting pathogens such as bacteria, viruses, and tumor cells (Hu et al., 2000). Macrophages produce large amounts of NO under the induction of specific cytokines and endotoxins, while NO can influence the synthesis and release of other cytokines ( $\mathrm{Li}$ et al., 2009). Macrophages confer immune protection, immune damage, and immune regulation by NO. A small amount of NO can relieve inflammation, while large amounts of synthetic NO can aggravate the inflammatory response. In the present study, the quantity of released $\mathrm{NO}$ after $72 \mathrm{~h}$ was lower on the titanium alloy surface than on the SS surface, but the difference was not statistically significant. Fewer macrophages adhered to the titanium alloy surface and the amount of NO released was lower than on the SS surface. NO released by macrophage activation exhibited a multiplicity function towards the inflammatory response, and it requires further examination.

MCP-1, also known as the mononuclear cell chemotaxis and activation factor, belongs to the $\beta$ family (Mishra et al., 2005; Ma et al., 2007). MCP-1 can specifically have a chemotaxis and activation effect on monocytes. MCP-1 can attract monocytes to infiltrate tissue through chemotaxis and plays an important role in macrophage accumulation in the damaged area (Anderson, 2004; Refai et al., 2004). Activated monocytes and macrophages can further produce chemokines such as MCP-1, inducing additional macrophage activation and accumulation. When MCP-1 mRNA expression is relatively high, it can be used as a standard to evaluate material biocompatibility. In our study, macrophages stimulated by SS exhibited significantly higher MCP-1 levels than those stimulated by the titanium alloy; additionally, fewer macrophages were observed on the titanium alloy surface. This indicates that the titanium alloy induces a lower degree of inflammation compared to that induced by SS.

TGF- $\beta 1$ is an important anti-inflammatory factor that can regulate immunosuppression and the inflammatory response (Lei et al., 2014; Yang et al., 2014). It plays a role in wound healing and local inflammatory reactions. Our results showed that TGF- $\beta 1$ mRNA expression was significantly higher on the titanium alloy surface than on the SS surface at 12 and $36 \mathrm{~h}$. This indicates that TGF- $\beta 1$ overexpressed in macrophages during the early stage on the titanium alloy surface. This may be because the implant material stimulated macrophage to produce TGF- $\beta 1$ for anti-inflammation soon after implanting the material into the body. Higher TGF- $\beta 1$ expression showed a better anti-inflammation effect for the titanium alloy than for the SS material.

In summary, macrophages participated in the inflammatory response by regulating $\mathrm{NO}$, MCP-1, and TGF- $\beta 1$ after implantation of dental materials. Our results showed that the macrophage count on the titanium alloy surface was lower than that on the SS, indicating a lower level of inflammation and better biocompatibility of the titanium alloy compared to the SS material.

\section{ACKNOWLEDGMENTS}

Research supported by the Army Logistics Research Project (\#BWS12J078).

\section{REFERENCES}

Anderson JM (2004). Inflammation, wound healing, and the foreign body response. In: Biomaterial Science: An Introduction to Materials Science in Medicine. Academic Press, Waltham, 296-304. 
Arango Duque G and Descoteaux A (2014). Macrophage cytokines: involvement in immunity and infectious diseases. Front. Immunol. 5: 491.

Cachinho SC, Pu F and Hunt JA (2013). Cytokine secretion from human peripheral blood mononuclear cells cultured in vitro with metal particles. J. Biomed. Mater. Res. A 101: 1201-1209.

Dalal A, Pawar V, McAllister K, Weaver C, et al. (2012). Orthopedic implant cobalt-alloy particles produce greater toxicity and inflammatory cytokines than titanium alloy and zirconium alloy-based particles in vitro, in human osteoblasts, fibroblasts, and macrophages. J. Biomed. Mater. Res. A 100: 2147-2158.

De Nardo L, Raffaini G, Ebramzadeh E and Ganazzoli F (2012). Titanium oxide modeling and design for innovative biomedical surfaces: a concise review. Int. J. Artif. Organs 35: 629-641.

Hu Y, Peng D and Huang W (2000). The dynamic changes of postburn complement activation and its effects on the secretory function of macrophages in severely burned mice. Zhonghua Shao Shang Za Zhi 16: 231-233.

Hu J, Jo M, Eastman BM, Gilder AS, et al. (2014). uPAR induces expression of TGF-beta and interleukin 4 in cancer cells to promote tumor-permissive conditioning of macrophages. Am. J. Pathol. 184: 3384-3393.

Jiang Y, Jia T, Gong W, Wooley PH, et al. (2013). Titanium particle-challenged osteoblasts promote osteoclastogenesis and osteolysis in a murine model of periprosthestic osteolysis. Acta Biomater. 9: 7564-7572.

Jin G, Cao H, Qiao Y, Meng F, et al. (2014). Osteogenic activity and antibacterial effect of zinc ion implanted titanium. Colloids Surf. B Biointerfaces 117: 158-165.

Lee J, Hurson S, Tadros H, Schüpbach P, et al. (2012). Crestal remodelling and osseointegration at surface-modified commercially pure titanium and titanium alloy implants in a canine model. J. Clin. Periodontol. 39: 781-788.

Lei L, Li HX, Huang MX, Chen S, et al. (2014). Hyperlipidemia inhibited inflammatory response to Porphyromonas gingivalis in apolipoprotein E knockout mice. Shanghai Kou Qiang Yi Xue 23: 409-412.

Li G, Yang P, Wang J, Zhao A, et al. (2009). An experimental study on the cytokine expression of macrophage influenced by biomaterials. Sheng Wu Yi Xue Gong Cheng Xue Za Zhi 26: 105-109.

Ma Y, Ren S, Pandak WM, Li X, et al. (2007). The effects of inflammatory cytokines on steroidogenic acute regulatory protein expression in macrophages. Inflamm. Res. 56: 495-501.

Malik AA, Radhakrishnan N, Reddy K, Smith AD, et al. (2002). Tubular cell-Escherichia coli interaction products modulate migration of monocytes through generation of transforming growth factor-beta and macrophage-monocyte chemoattractant protein-1. J. Endourol. 16: 599-603.

Manzanares MC, Franch J, Carvalho P, Belmonte AM, et al. (2001). BS-SEM evaluation of the tissular interactions between cortical bone and calcium-phosphate covered titanium implants. Bull. Group Int. Rech. Sci. Stomatol. Odontol. 43: 100-108.

Mishra L, Derynck R and Mishra B (2005). Transforming growth factor-beta signaling in stem cells and cancer. Science 310: 68-71.

Refai AK, Textor M, Brunette DM and Waterfield JD (2004). Effect of titanium surface topography on macrophage activation and secretion of proinflammatory cytokines and chemokines. J. Biomed. Mater. Res. A 70: 194-205.

Steflik DE, Corpe RS, Young TR, Sisk AL, et al. (1999). The biologic tissue responses to uncoated and coated implanted biomaterials. Adv. Dent. Res. 13: 27-33.

Yang X, Wei H, Qin L, Zhang S, et al. (2014). Reciprocal interaction between fish TGF-beta1 and IL-1beta is responsible for restraining IL-1beta signaling activity in grass carp head kidney leukocytes. Dev. Comp. Immunol. 47: 197-204.

Yazici H, Fong H, Wilson B, Oren EE, et al. (2013). Biological response on a titanium implant-grade surface functionalized with modular peptides. Acta Biomater. 9: 5341-5352. 